\title{
Electron Holographic Li-ion Profiling of an Inorganic Solid-state Electrolyte
}

\author{
T. Hirayama*, K. Yamamoto*, T. Asaka*, A. Kuwabara*, Y. Iriyama**, and Z. Ogumi*** \\ *Nanostructures Research Laboratory, Japan Fine Ceramics Center, Nagoya 456-8587, Japan \\ ** Department of Materials Science and Engineering, Faculty of Engineering, Shizuoka University, \\ Hamamatsu, Shizuoka 431-8561, Japan \\ ***Innovation Collaboration Center, Kyoto University, Kyoto 615-8520, Japan
}

Lithium ion batteries (LIBs) can efficiently store and release large amount of electricity, and are considered the most promising devices for reducing $\mathrm{CO}_{2}$ emissions [1]. However, the distribution and diffusion of $\mathrm{Li}$ ions which control the performance of the batteries have not yet been studied sufficiently. These days we are trying to observe lithium ion distributions in all-solid-state LIBs, and here we report our first in-situ Li-ion profiling experiment for an inorganic solid-state electrolyte.

A $90 \mu \mathrm{m}$ thick ceramic sheet of composition $\mathrm{Li}_{1+\mathrm{x}+\mathrm{y}} \mathrm{Al}_{\mathrm{y}} \mathrm{Ti}_{2-\mathrm{y}} \mathrm{Si}_{\mathrm{x}} \mathrm{P}_{3-\mathrm{x}} \mathrm{O}_{12}$ (ionic conductivity of $10^{-4} \mathrm{~S}$ $\mathrm{cm}^{-1}$ at room temperature; OHARA Inc., Japan) was used as the solid electrolyte. Electrodes of crystalline $\mathrm{LiCoO}_{2}$ were deposited on both sides of the sheet by pulsed laser deposition. Then, one side was coated with gold and the other side with platinum. This sample, with an electron-beam transparent thin region prepared by focused ion beam (FIB) milling, was loaded on a purpose-built TEM holder equipped with two fixed electrodes for applying voltage.

Figure 1 shows a schematic of the experiment and a typical phase map when a voltage of $3.8 \mathrm{~V}$ was applied to the sample. The bright contrast in Fig. 1(b) shows the high electric potential formed by the positive Li ions. Figure 2 displays a schematic of the experiment and a phase map when two electrodes of the sample were connected without a potential difference applied, showing that the electric potential in the electrolyte is almost flat in the observed region.

In conclusion, we have succeeded in clearly profiling the Li-ion distribution in a solid-state electrolyte. We believe that this technique will enable us to reveal essential features of electrochemical reactions in $\mathrm{Li}$-ion batteries and contribute to the development of superior Li-ion batteries.

The authors would like to thank Dr. Craig Fisher of Japan Fine Ceramics Center for informative discussions. This work was done as part of the Research \& Development Initiative for Scientific Innovation of New Generation Batteries (RISING) project of the New Energy and Industrial Technology Development Organization (NEDO).

Reference

[1] M. Armand and J.-M. Tarascon, Nature 451, (2008) 652. 


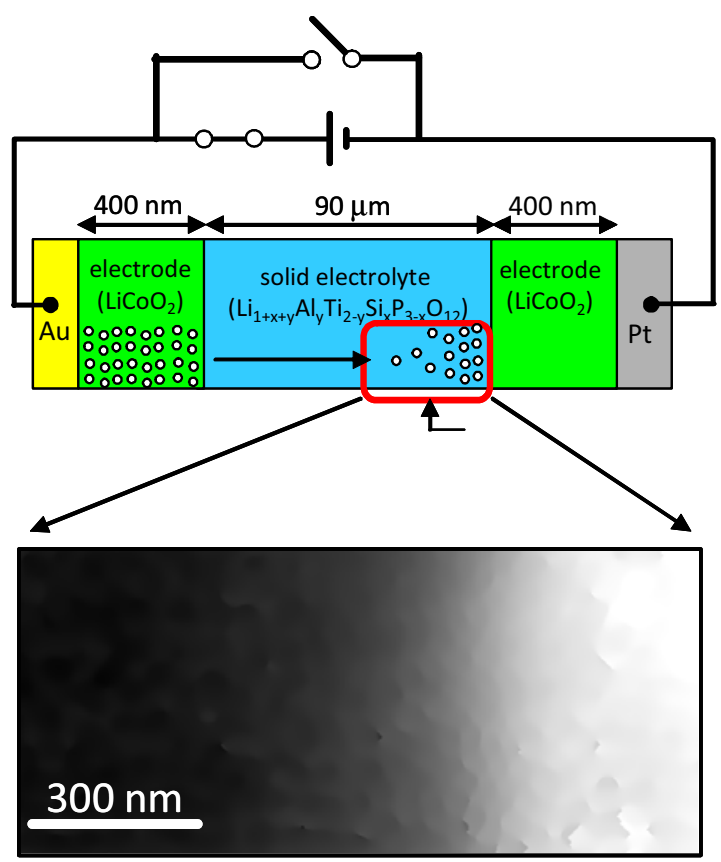

FIG. 1. In-situ measurement of Li-ion distribution when a voltage of $3.8 \mathrm{~V}$ was applied between $\mathrm{Au}$ and Pt electrodes: (a) schematic of the experiment, and (b) phase map. The bright contrast in (b) indicates high electric potential from positive $\mathrm{Li}$ ions.

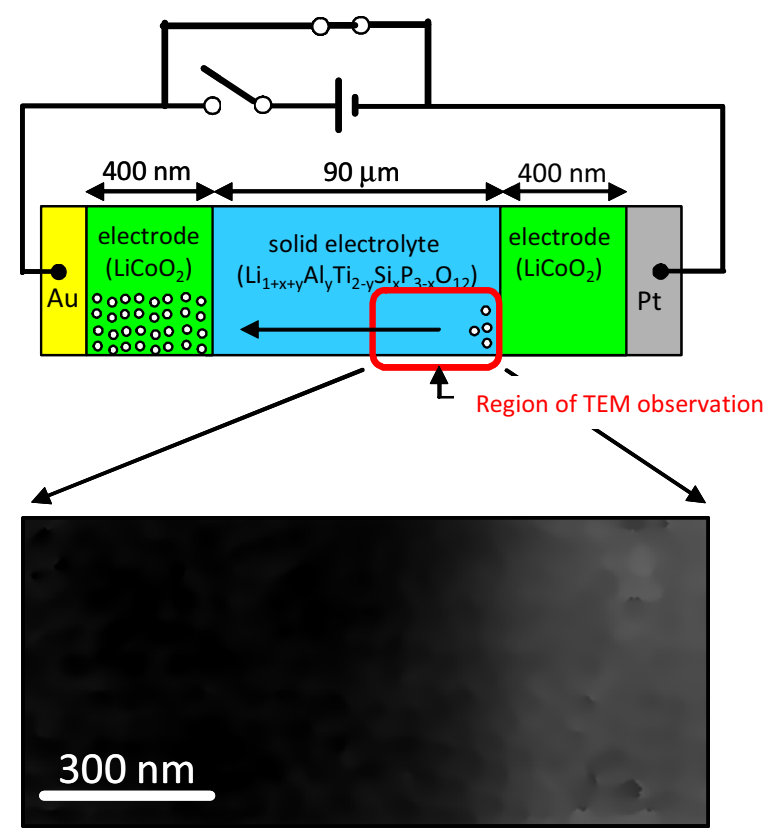

FIG. 2. In-situ measurement of Li-ion distribution when the two electrodes were short-circuited: (a) schematic of the experiment, and (b) phase map. The electric potential in (b) is almost flat across the observed region. 